\title{
Real-Time Travel Mode Detection with Smartphone Sensing and Machine Learning
}

\author{
Elton F. de S. Soares, Carlos Alberto V. Campos, Sidney Cunha de Lucena \\ ${ }^{1}$ Universidade Federal do Estado do Rio de Janeiro \\ Programa de Pós-Graduação em Informática - (PPGI-UNIRIO) \\ Rio de Janeiro - RJ - Brazil \\ \{elton.soares, beto, sidney\}@uniriotec.br
}

\begin{abstract}
The detection of the travel modes used and, the prediction of trip purposes, through smartphone sensors data have emerged as two research challenges in recent years. Both of these problems have been deeply investigated in isolation, while the problem of inferring mode and purpose at the same time and, more specifically, using the same preprocessing algorithm has been less explored. Also, few studies presented solutions that can execute the detection of user travel modes in real-time, and even fewer have presented the evaluation of these solutions in a realistic manner. Meanwhile, some of the previous studies claim that off-the-shelf activity recognition solutions, do not perform well in the travel mode detection task, although many of them do not present a quantitative evidence of their bad performance. Thus, in this work, we propose three techniques for real-time travel mode detection, using different combinations of smartphone sensors, and one technique for join travel mode detection and trip purpose prediction using a single preprocessing algorithm, in real-time. We empirically evaluated the proposed techniques and an off-the-shelf activity recognition solution using field tests and cross-validation experiments with private and public mobility datasets.
\end{abstract}

\section{Problem Characterization and Motivation}

The identification of users' travel modes (i.e., modes of transportation) through their smartphone sensors is a growing topic of research, with many applications in the field of Smart Mobility and Internet of Things (IoT), specially with respect to ITS [Soares 2019].

In particular, online (i.e., real-time) travel mode detection can provide contextawareness, which is useful for Location-Based Services (LBS) [Prelipcean et al. 2017], in order to customize information delivery based on users' needs and possibilities of interaction. It may also be applied for offline detection use cases, depending on the frequency in which the travel mode information needs to be available for the final application.

Among the several features that may be used to describe mobility patterns, the travel mode and trip purpose specifically contribute to their better understanding, thus benefiting the generation of automatic (or semi-automatic) travel diaries [Nitsche et al. 2014], recommendation systems, personal assistants and other mobile data collection applications [Schobel et al. 2017].

Detecting which mode of transportation and trip purpose are being used by citizens through their smartphone sensors is a hard task, if we consider all the range of possible transportation modes and trip purposes available in urban centers. There are many 
commercial solutions that tackle instances of these problems using historical information from user mobility behaviour and/or rule-based classification schemes, like Google Maps Timeline ${ }^{1}$, for example.

These solutions do not provide, however, a generic technique that can be used to infer the mode and the purpose of user trips using only a small amount of user data and without requiring a connection to a cloud server to process the information captured through smartphone sensors. Besides, most of these solutions are able to classify only a subset of all the modes and purposes that can be inferred from user mobility data, limiting their usefulness for a broader range of use cases.

\subsection{Objectives and Contributions}

This work's main objective is to develop solutions for the problems of real-time travel mode detection and trip purpose prediction. These solutions must be able to identify the mode of transportation and the purpose of the trips made by smartphone users, in realtime, with a high accuracy and low resource consumption.

The accuracy of this identification is essential for the context-aware applications that might use these contextual-information for delivering customized messages and services to the user. Also, ITS applications may use the inferred travel modes and trip purposes for infrastructure management and adaptation, which makes precision and recall of those inferences crucial for their success.

The low resource consumption is another critical requirement of the ideal solution, because it cannot consume a high proportion of the computational resources available on the smartphone, as these are already disputed by the other applications and services. Besides, smartphone resources might be very limited, depending on the model and vendor. Therefore, the optimal solution should be able to run on any smartphone device compatible with the context-aware applications it might serve.

The present work will try to answer the following research questions:

1. Can off-the-shelf activity recognition solutions perform real-time travel mode detection satisfactorily with regards to accuracy, precision and recall of the inferences made?

2. Is detecting a person's mode of transportation through statistical features extracted from time series of smartphone location sensors measurements, in real-time, truly possible?

3. How can we detect the mode of transportation and the purpose of trips, in realtime, using statistical features extracted from time series of smartphone location sensors measurements?

4. Can automated machine learning help us generate more accurate classification models for real-time travel mode detection based on smartphone sensor measurements?

5. Does the use of dimensionality reduction techniques upon the features extracted from the time series of multiple smartphone sensor measurements reduce the cost of training and running travel mode classifiers without substantially reducing accuracy, precision and recall?

\footnotetext{
${ }^{1}$ https://www.google.com.br/maps/timeline
} 
6. Do higher order statistical features of the time series of smartphone sensors measurements, allow a better identification of travel modes, in real-time, with regards to accuracy, precision and recall?

7. Can recurrent neural networks be used to generate more efficient real-time travel mode detection models than other state-of-the-art machine learning techniques, with regards to classification accuracy and model size trade-off?

The main scientific contributions of this work are:

1. A real-time travel mode detection technique that uses features extracted from time series of smartphone GPS, WiFi and Cellular Network sensors measurements and supervised machine learning algorithms to train travel mode classification models that rely only on smartphone hardware resources, without the need of internet connectivity.

2. A prototype implementation of the proposed technique as a mobile application on Android platform, namely CityTracks-RT, and the experimental analysis of in the field performance of the proposed solution in the metropolitan region of Rio de Janeiro.

3. A mobile application for multi-sensor data collection and real-time travel mode detection, namely CityTracks-AWARE, using an off-the-shelf activity recognition solution and the experimental analysis of in the field performance of the solution in the metropolitan area of Rio de Janeiro.

4. A method for building real-time travel mode detection and trip purpose prediction models using features extracted from time series of smartphone location measurements and the experimental analysis of the proposed method based on quantitative metrics obtained through cross-validation using real smartphone data, collected during CityTracks-RT evaluation experiments.

5. An experimental analysis of the improvement obtained in real-time travel mode detection performance, when using automated machine learning techniques, based on quantitative metrics obtained through cross-validation using real smartphone data from a public travel survey dataset.

6. A real-time travel mode detection method based on recurrent neural networks and features extracted from time series of multiple smartphone sensor measurements, namely TMD-LSTM, and the experimental analysis of the proposed technique based on quantitative metrics obtained through cross-validation using real smartphone data.

\section{Related Work}

Travel mode detection via smartphones can use a wide variety of sensors, however, most state-of-the-art solutions found use only GPS data [Xiao et al. 2015, Bantis and Haworth 2017, Wang et al. 2017, Mäenpää et al. 2017, Dabiri and Heaslip 2018]. Some solutions used accelerometer readings and cellular data to compensate for GPS signal losses [Quintella et al. 2016, Martin et al. 2017] and others used data from the gyroscope, magnetometer, and other less conventional sensors such as barometer and gravity sensor to reduce power consumption [Bedogni et al. 2016, Su et al. 2016, Chen et al. 2017, Shafique and Hato 2016, Fang et al. 2016, Assemi et al. 2016]. To the best of our knowledge, none of the related 
work presented the evaluation of an application for real-time travel mode detection in a real use scenario.

In [Ermagun et al. 2017] authors propose the use of online location-based search and discovery services, such as Google Places API to improve trip purpose prediction. They apply nested logit and random forest to identify five trip purposes from trips collected during the 2010 Travel Behaviour Inventory in Minneapolis-St. Paul metropolitan area. Other works in this topic explored semi-automated trip purpose inference through participatory sensing [Seo et al. 2017], combination of GPS trajectories, POIs and social media data [Meng et al. 2017], data selection from different seasons for model training and testing [Gong et al. 2017], trip purpose detection with artificial neural networks with particle swarm optimization[Xiao et al. 2016].

In comparison with individual travel mode detection and trip purpose prediction, fewer works proposed techniques that could simultaneously perform both tasks. One of the earliest works to present that type of solution [Stopher et al. 2005] proposed the use of heuristics based on features extracted from GPS logs, such as average speed and maximum speed, combined with GIS information, such as street lines and public transportation stops, to identify the travel modes and purposes of trips made by citizens in Sidney, Australia. The main disadvantage of their technique is that it is highly dependent on GIS information, which might not always be available, accurate and up to date.

The latest solution was proposed in CTASS [Batool et al. 2017] and consisted of a digital framework for contextualized travel behaviour advice to cardiac patients that utilized the Moves app [Evenson and Furberg 2017] for non-motorized travel mode detection in conjunction of its Health Travel Behaviour (HTB) app that performed trip purpose identification and trip data collection. This work, however, did not detail which features and classification models were used for each detection task, neither presented a quantitative evaluation of its performance.

\section{Results}

As a result of this thesis we developed a technique that allows detecting the travel mode of smartphone users, in real time. This technique was implemented on a prototype Android application, named CityTracks-RT, that used supervised machine learning models. This prototype application was published in Google Play app store ${ }^{2}$ and it's source code was published under MIT license at GitHub ${ }^{3}$. The performance of the prototype was evaluated through the analysis of quantitative metrics obtained via field tests with 37 volunteers in Rio de Janeiro and the results indicate that the proposed technique is capable of detecting the travel modes of smartphone users in urban centers. We have also published the prototype application that was used to execute the additional evaluation experiments with an off-the-shelf activity recognition solution, namely CityTracks-AWARE, on both GitHub $^{4}$ and Google Play ${ }^{5}$.

Moreover, a hybrid solution for real-time travel mode detection and trip purpose prediction was developed. This solution uses a single preprocessing algorithm to extract

\footnotetext{
${ }^{2}$ https://play.google.com/store/apps/details?id=unirio.citytracksrt

${ }^{3}$ https://github.com/eltonfss/CityTracks-RT

${ }^{4}$ https://github.com/eltonfss/CityTracks-AWARE

${ }^{5} \mathrm{https}$ ://play.google.com/store/apps/details?id=citytracksaware.client
} 
features that are used to train classification models through supervised ML algorithms. Its performance was evaluated through the analysis of quantitative metrics obtained via k-fold cross-validation. The maximum accuracy reached for travel mode detection and trip purpose prediction was $88 \%$ and $81 \%$, respectively. Therefore, it can be concluded that the proposed solution is capable of detecting the travel mode and predict the trip purpose of a trip using only 60 seconds of smartphone collected location data, allowing context-aware information systems to use this contextual information to provide personalized services and better predict user behaviour with respect to mobility patterns and transportation preferences.

Both techniques are illustrated in Figure 1.

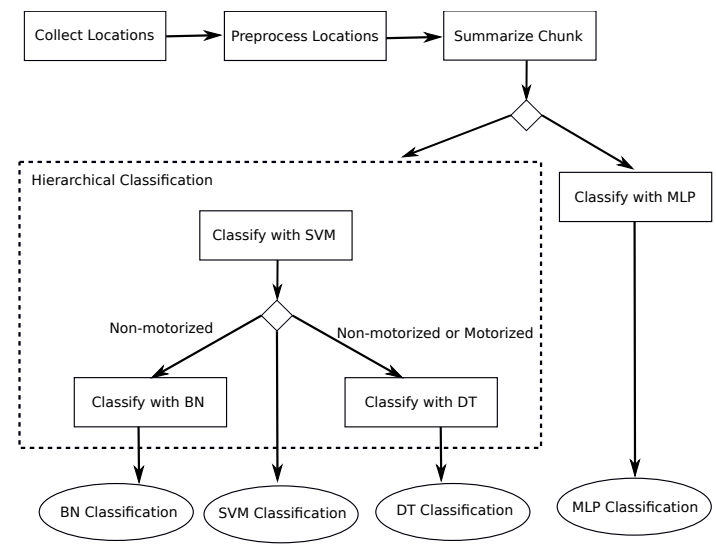

(a)

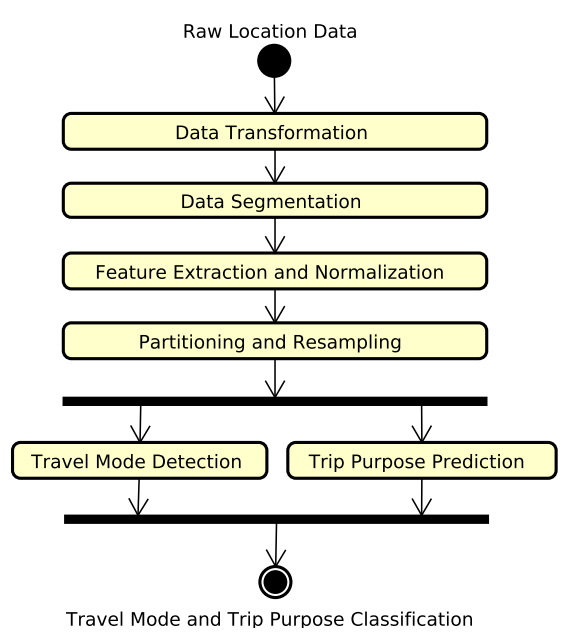

(b)

Figure 1. (a) Proposed Real-Time Travel Mode Detection Solution. *MLP - Multilayer Perceptron, SVM - Support Vector Machine, BN - Bayesian Net, DT - Decision Table; (b) Overview of travel mode detection and trip purpose prediction solution.

We also evaluated the use of AutoML and feature engineering techniques to enhance travel mode detection with multiple sensors. The evaluation experiments showed that these methods can greatly improve classification accuracy, reaching a maximum of 97\% accuracy, precision, recall and f-measure on the TMD Dataset using the AutoSklearn Global Optimization framework. This is the first work to present evidence that AutoML frameworks can outperform human researchers in the combined algorithm selection and hyperparameter optimization of ML classifiers for the travel mode detection problem. The source code of this experiment was also published on GitHub under MIT license ${ }^{6}$.

Finally, we developed TMD-LSTM, a technique that uses RNNs to learn travel mode patterns through multiple sensing modalities, and its online detection capabilities were evaluated using a public benchmark dataset. The experimental results obtained indicate that the method proposed is capable of detecting modes with up to $90 \%$ accuracy and a lower memory consumption and computational cost than other ML approaches with comparable performance. The source code of this implementation was published in the form of a generic travel mode detection library that can be reused and extended by other

\footnotetext{
${ }^{6}$ https://github.com/eltonfss/TMDAutoML
} 
researchers on GitHub ${ }^{7}$.

Figure 2 illustrates both approaches to travel mode detection.

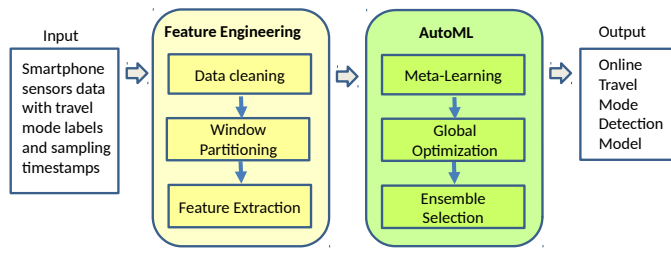

(a)

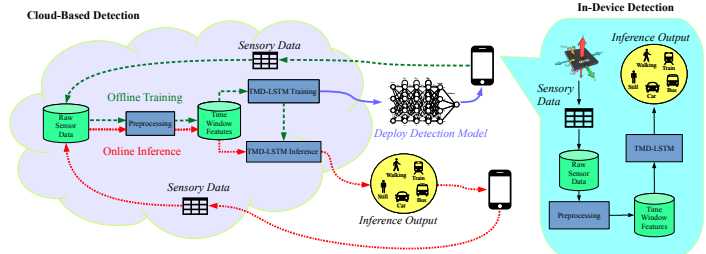

(b)

Figure 2. (a) Components of the proposed method for building real-time travel mode detection models; (b) Cloud-based and In-device real-time travel mode detection with TMD-LSTM.

For a more detailed description of the solutions and the results obtained in their experimental evaluation, please refer to the the master's thesis [Soares 2019].

\section{Impact}

As a result of this master's thesis we have published 3 conference papers, including a publication at IEEE VTC 2017 and one at IEEE GLOBECOM 2019, two major venues for discussing innovative ideas in Vehicular Technology, Computer Networks and Wireless Communication, respectively. The list of conference publications follows:

- Soares, Elton F. de S., Quintella, Carlos Álvaro de M. S., and Campos, Carlos Alberto V. "CityTracks-RT: Uma aplicação para detecção do modo de transporte em tempo real nos centros urbanos”. In SBSI, pp. 222-229, May 2017 (Qualis B2).

- Soares, Elton F. de S., Quintella, Carlos Álvaro de M. S., and Campos, Carlos Alberto V. "Towards an application for real-time travel mode detection in urban centers". In IEEE VTC, pp. 1-5, Sep. 2017. (Qualis B1)

- Soares, Elton F. de S., Salehinejad, Hojjat, Campos, Carlos Alberto V., and Valaee, Shahrokh. "Recurrent neural networks for online travel mode detection". In IEEE GLOBECOM, Dec. 2019. (Qualis A1) review:

In addition, we have also published 2 journal papers and 1 journal paper under

- Soares, Elton F. de S., Revoredo, Kate, Quintella, Carlos Álvaro de M. S., Campos, Carlos Alberto V., and Baião, Fernanda. "A combined solution for real-time travel mode detection and trip purpose prediction". In IEEE Transactions on Intelligent Transportation Systems, vol 20, pp. 4655-4664, May 2019. (Qualis A1)

- Soares, Elton F. de S., Campos, Carlos Alberto V., and de Lucena, Sidney Cunha. "Online travel mode detection method using automated machine learning and feature engineering". In Future Generation Computer Systems, vol 101, pp. 12011212, Dec. 2019. (Qualis A2)

\footnotetext{
${ }^{7}$ https://github.com/eltonfss/TMDLibrary
} 
- Soares, Elton F. de S., Quintella, Carlos Álvaro de M. S., Campos, Carlos Alberto V. "Smartphone-based Real-Time Travel Mode Detection for Intelligent Transportation Systems" is currently on the 2nd round of reviews for publication in IEEE Transaction on Vehicular Technology (Qualis A1)

Moreover, in the context of this master's thesis, we also co-authored the following conference paper:

- Teteo, Leonardo, Moura, Pedro Nuno, Soares, Elton F. de S., Campos, Carlos Alberto V. "Um Framework de Extração e Etiquetamento de Informações de Trânsito”. In WPerformance, July 2019. (Qualis B3)

\section{References}

Assemi, B., Safi, H., Mesbah, M., and Ferreira, L. (2016). Developing and validating a statistical model for travel mode identification on smartphones. IEEE Transactions on Intelligent Transportation Systems, 17(7):1920-1931.

Bantis, T. and Haworth, J. (2017). Who you are is how you travel: A framework for transportation mode detection using individual and environmental characteristics. Transportation Research Part C: Emerging Technologies, 80:286-309.

Batool, T., Vanrompay, Y., Neven, A., Janssens, D., and Wets, G. (2017). Ctass: a framework for contextualized travel behavior advice to cardiac patients. Procedia Computer Science, 113:303-309.

Bedogni, L., Di Felice, M., and Bononi, L. (2016). Context aware android applications through transportation mode detection techniques. Wireless Communications and Mobile Computing, 16(16).

Chen, K.-Y., Shah, R. C., Huang, J., and Nachman, L. (2017). Mago: Mode of transport inference using the hall-effect magnetic sensor and accelerometer. Proc. ACM Interact. Mob. Wearable Ubiquitous Technol., 1(2):8:1-8:23.

Dabiri, S. and Heaslip, K. (2018). Inferring transportation modes from gps trajectories using a convolutional neural network. Transportation Research Part C: Emerging Technologies, 86:360-371.

Ermagun, A., Fan, Y., Wolfson, J., Adomavicius, G., and Das, K. (2017). Real-time trip purpose prediction using online location-based search and discovery services. Transportation Research Part C: Emerging Technologies, 77:96-112.

Evenson, K. R. and Furberg, R. D. (2017). Moves app: a digital diary to track physical activity and location. Br J Sports Med, 51(15):1169-1170.

Fang, S.-H., Liao, H.-H., Fei, Y.-X., Chen, K.-H., Huang, J.-W., Lu, Y.-D., and Tsao, Y. (2016). Transportation modes classification using sensors on smartphones. Sensors, 16(8):1324.

Gong, L., Kanamori, R., and Yamamoto, T. (2017). Data selection in machine learning for identifying trip purposes and travel modes from longitudinal gps data collection lasting for seasons. Travel Behaviour and Society.

Mäenpää, H., Lobov, A., and Lastra, J. L. M. (2017). Travel mode estimation for multimodal journey planner. Transportation Research Part C: Emerging Technologies, $82: 273-289$. 
Martin, B. D., Addona, V., Wolfson, J., Adomavicius, G., and Fan, Y. (2017). Methods for real-time prediction of the mode of travel using smartphone-based gps and accelerometer data. Sensors, 17(9):2058.

Meng, C., Cui, Y., He, Q., Su, L., and Gao, J. (2017). Travel purpose inference with gps trajectories, pois, and geo-tagged social media data. In Big Data (Big Data), 2017 IEEE International Conference on, pages 1319-1324. IEEE.

Nitsche, P., Widhalm, P., Breuss, S., Brändle, N., and Maurer, P. (2014). Supporting largescale travel surveys with smartphones-a practical approach. Transportation Research Part C: Emerging Technologies, 43:212-221.

Prelipcean, A. C., Gidófalvi, G., and Susilo, Y. O. (2017). Transportation mode detection - an in-depth review of applicability and reliability. Transport Reviews, 37(4):442464.

Quintella, C. A. d. M. S., Andrade, L. C., and Campos, C. A. v. (2016). Detecting the transportation mode for context-aware systems using smartphones. In IEEE Intelligent Transportation Systems (ITSC).

Schobel, J., Pryss, R., Schlee, W., Probst, T., Gebhardt, D., Schickler, M., and Reichert, M. (2017). Development of mobile data collection applications by domain experts: Experimental results from a usability study. In International Conference on Advanced Information Systems Engineering, pages 60-75. Springer.

Seo, T., Kusakabe, T., Gotoh, H., and Asakura, Y. (2017). Interactive online machine learning approach for activity-travel survey. Transportation Research Part B: Methodological.

Shafique, M. A. and Hato, E. (2016). Travel mode detection with varying smartphone data collection frequencies. Sensors, 16(5).

Soares, E. F. d. S. (2019). Real-Time Travel Mode Detection with Smartphone Sensing and Machine Learning. Programa de Pósgraduação em Informática - PPGI, Universidade Federal do Estado do Rio de Janeiro - UNIRIO, 143 páginas.

Stopher, P. R., Jiang, Q., FitzGerald, C., et al. (2005). Processing gps data from travel surveys. 2nd international colloqium on the behavioural foundations of integrated land-use and transportation models: frameworks, models and applications, Toronto.

Su, X., Caceres, H., Tong, H., and He, Q. (2016). Online travel mode identification using smartphones with battery saving considerations. IEEE Transactions on Intelligent Transport. Systems, 17(10).

Wang, B., Gao, L., and Juan, Z. (2017). Travel mode detection using gps data and socioeconomic attributes based on a random forest classifier. IEEE Transactions on Intelligent Transportation Systems.

Xiao, G., Juan, Z., and Zhang, C. (2015). Travel mode detection based on gps track data and bayesian networks. Computers, Environment and Urban Systems, 54:14-22.

Xiao, G., Juan, Z., and Zhang, C. (2016). Detecting trip purposes from smartphonebased travel surveys with artificial neural networks and particle swarm optimization. Transportation Research Part C: Emerging Technologies, 71:447-463. 